\section{TRIANGULAR-ELEMENT FINITE-ELEMENT ANALYSIS OF A TRAPEZOID POLYMER OPTICAL WAVEGUIDE}

Shyh-Lin Tsao, ${ }^{1}$ Jingshown $\mathrm{Wu},{ }^{2}$ and Yue-Loong $\mathrm{Her}^{2}$

'Department of Electronic Engineering

St. John's and St. Mary's Institute of Technology

Tamsui, Taiwan

${ }^{2}$ Department of Electrical Engineering

National Taiwan University

Taipei, Taiwan, Republic of China

\section{Received 3 December 1996}

ABSTRACT: Because of mechanical properity, polymer optical waveguide interconnections can increase design flexibility. In this article we analyze the propagation characteristics of the trapezoid polymer optical waveguide by use of the finite-element method with triangular elements. The trapezoid polymer optical waveguide promises a larger cutoff frequency over the conventional rectangular waveguide. (C) 1997 John Wiley \& Sons, Inc. Microwave Opt Technol Lett 15: 87-89, 1997.

Key words: polymer optical waveguide; finite-element method

\section{INTRODUCTION}

The switching time of electronic devices and the chip integration scale have been improved greatly recently, and hard-wire transmission delays have become the crucial bottleneck on the system. Because the optical interconnection can meet the requirements of high density, high speed, and low cost for VLSI fabrication and replacement of electrical transmission line, it becomes increasingly important for high-speed computers or communication systems [1, 2]. At higher data rates (above $1 \mathrm{Gbps}$ ), as the lengths of interconnections within chip and from chip to chip increase (above $1 \mathrm{~mm}$ ), the optical interconnections have lower power dissipation [3].

In this Letter, we study the trapezoid and rectangular polymer optical waveguides for optical interconnections. The dispersion relation is analyzed by use of the finite-element method with triangular elements, which can provide the benefit of defining the tilt angle of the roof slope. In this work we find that the trapezoid waveguide can provide higher cutoff frequency than the rectangular waveguide with the same refractive indices and geometrical scale.

\section{TEFEM ANALYSIS}

Many microwave or optical waveguides have been analyzed with the use of the finite-element method (FEM). The triangular element finite-element method (TEFEM) has become especially popular for investigating nonrectangular waveguides $[4,5]$. A trapezoid optical waveguide and a rectangular-type waveguide, along with their geometrical parameters, are shown in Figures 1(a) and 1(b), respectively. The electromagnetic field propagating along the $z$-axis in the waveguide can be expressed as $\exp [-j(\beta z-\omega t)]$, where $z$ is the propagation direction, $\beta$ is the propagation constant in the $z$ direction, $\omega$ is the angular frequency, and $t$ is time. To apply the TEFEM, we discretize the cross section of the optical waveguide by suitable triangular-edged elements, as shown in Figure 2. The triangular element consists of three corners, whereas the local corners numbered 1-3 proceed counterclockwise for the $\mathrm{z}$-axis field $\phi_{z}$. The other three sides, labeled 4-6, represent the tangential component $\left(\phi_{t}\right.$ : in-

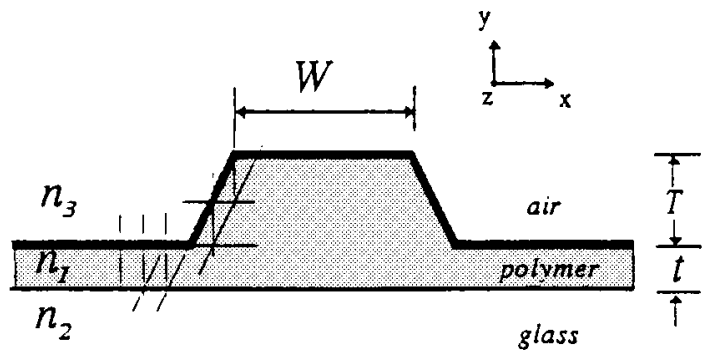

(a)

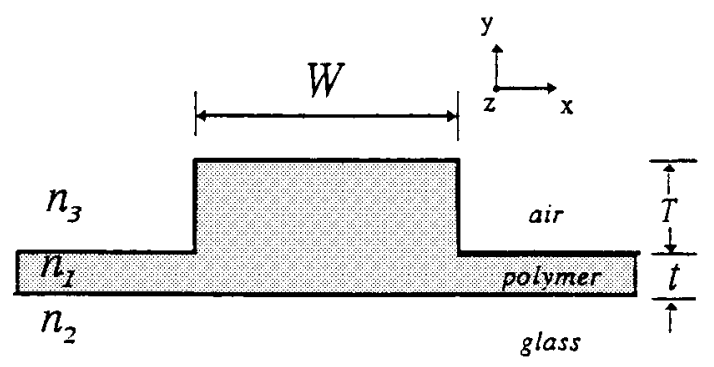

(b)

Figure 1 (a) Cross section of trapezoid waveguide, (b) Cross section of rectangular-type waveguide

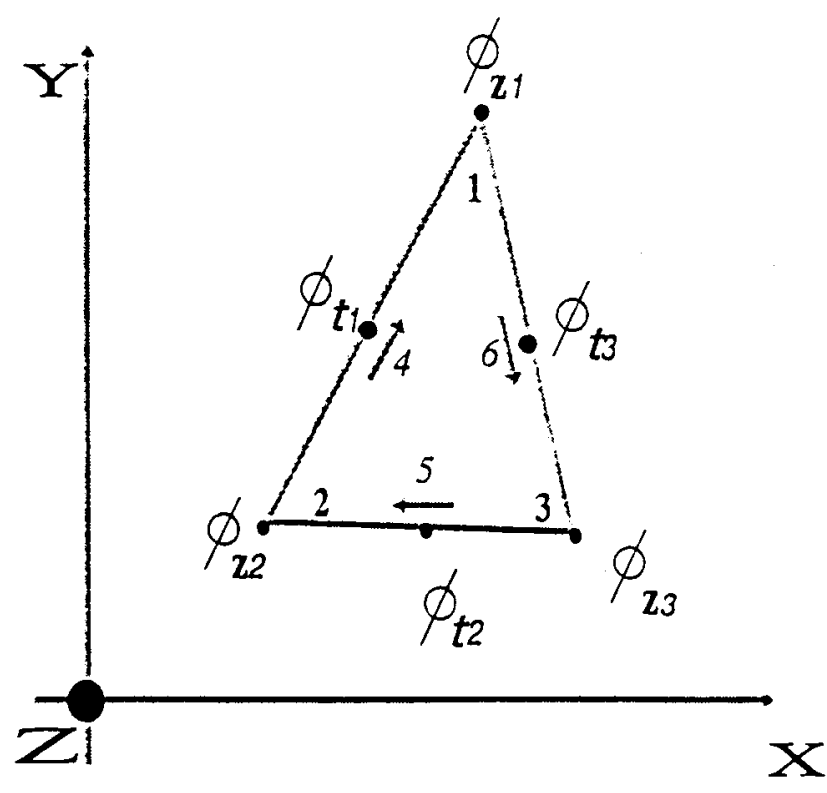

Figure 2 Schematic diagram of triangular elements

cludes both $x$-axis and $y$-axis field components). Assuming the optical waveguide to be isotropic and using Maxwell's equations, we can represent the vectorial Helmholtz equation as

$$
\begin{aligned}
& \nabla \times \nabla \times \Phi-k_{0}^{2} n^{2} \Phi=0 \\
& \Phi=\phi_{x} e_{x}+\phi_{y} e_{y}+\phi_{z} e_{z}
\end{aligned}
$$

where $\Phi$ denotes either $E$ or $H, k_{0}(=2 \pi / \lambda, \lambda$ is the wavelength) is the free-space wave number, and $n$ is the refractive index of the polymer waveguide. With the use of the variation method to solve the partial differential equa- 
tions [6], we derived the functional for Eq. (1) as

$$
F=\iint_{\Omega}\left[(\nabla \times \Phi)^{*} \cdot(\nabla \times \Phi)-k_{0}^{2} n^{2} \Phi^{*} \cdot \Phi\right] d x d y .
$$

Here the asterisk denotes the complex conjugate and $\Omega$ is the waveguide cross-section area where the integration is performed over it. The optical transverse components $\phi_{x}, \phi_{y}$, and axial component $\phi_{z}$ of the electromagnetic field vector $\Phi$ can be approximately represented as three first-order polynomials [5] in $x$ and $y$, respectively, such that

$$
\begin{aligned}
\phi_{x} & =\left[S_{x}\right]^{T}\left[\phi_{t}\right]_{e}, \\
\phi_{y} & =\left[S_{y}\right]^{T}\left[\phi_{t}\right]_{e}, \\
\phi_{z} & =\left[S_{z}\right]^{T}\left[\phi_{z}\right]_{e},
\end{aligned}
$$

where $\left[S_{x}\right],\left[S_{y}\right],\left[S_{z}\right]$ are the shape function vectors for the triangular edge elements, the superscript $T$ denotes the inverse matrix, and $\left[\phi_{z}\right]_{e}$ and $\left[\phi_{t}\right]_{e}$ are the $z$-axis field vector and the edge tangential-field vector for each element, respectively. The coordinate relation between the transverse components $\phi_{x}, \phi_{y}$ and tangential component $\phi_{t}$ can be generally expressed as

$$
\phi_{t}=\phi_{x} \cos \theta+\phi_{y} \sin \theta,
$$

where $\theta$ is the tilt angle between the $x$ axis and the edge of $\phi_{t}$. If we substitute Eq. (3) into Eq. (2), apply the variational principle, and then solve the eigenvalue equations to get the propagation constant $\beta$, we can calculate the normalized refractive index $b$ from the following equation:

$$
b=\frac{\left(\beta / k_{0}\right)^{2}-n_{2}^{2}}{n_{1}^{2}-n_{2}^{2}},
$$

where $n_{1}$ and $n_{2}$ are the refractive indices of the polymer waveguide and silica, respectively. The normalized frequency $\nu$ is defined as

$$
\nu=(T+t) / \lambda,
$$

where $T+t$ is the thickness of the trapezoid polymer waveguide.

\section{EXPERIMENTAL AND NUMERICAL RESULTS}

We fabricate a trapezoid polymer optical waveguide on silica substrate by using an embossing method with the Si mold, which is prepared by micromachining. The cross section of the fabricated trapezoid polymer optical waveguide using PMMA $\left(n_{1}=1.491\right)$ on silica substrate $\left(n_{2}=1.453\right)$ is shown in Figure 3. Because of anisotropic etching on the Si wafer, the tilt angle is $54.7^{\circ}$. We apply TEFEM to analyze the propagation characteristics of the trapezoid waveguide $(\theta=$ $\left.54.7^{\circ}\right)$ and the rectangular waveguide $\left(\theta=90^{\circ}\right)$. The numerical result is shown in Figure 4, the cutoff frequency of the trapezoid waveguide is located at $\nu=1.5$, which is larger than the cutoff frequency of the rectangular waveguide $\nu=$ 1.3. By selecting small $\Delta n\left(\Delta n=n_{1}-n_{2}\right)$ and large $\Delta t$ $(\Delta t=T-t)$, we can increase the cutoff frequency of the waveguide. Also, we find that the trapezoid optical waveguide promises a larger cutoff frequency over the conventional rectangular waveguide.

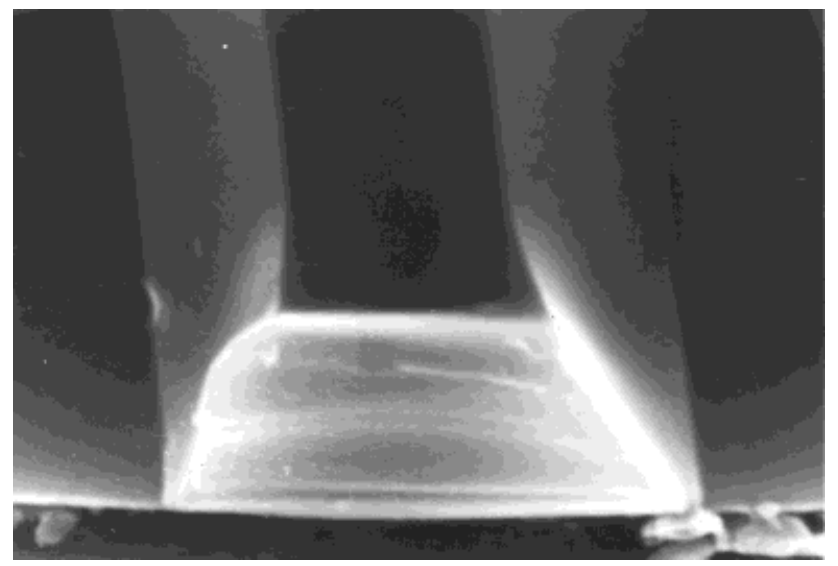

Figure 3 SEM micrograph of the cross section of trapezoid polymer optical waveguide

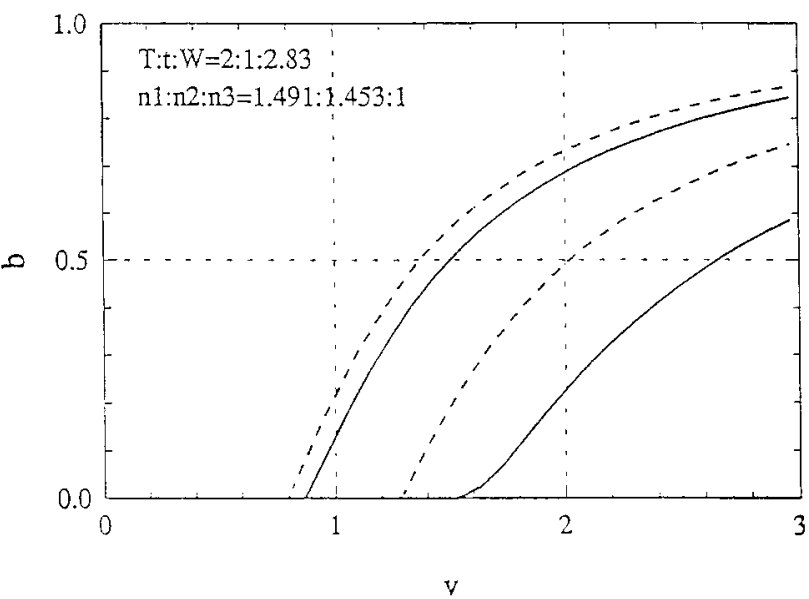

Figure 4 Comparison of propagation characteristics between optical trapezoid waveguide and rectangular waveguides. Solid lines, trapezoid waveguide; dashed lines, rectangular waveguide

\section{CONCLUSIONS}

Due to large bandwidth and minimal cross talk, the polymer optical interconnection will play an important role in optical interconnections in the future. The cheap organic polymer optical waveguide has presented great potential. In this article we use the TEFEM method to analyze the propagation characteristics of a trapezoid polymer optical waveguide.

The results show that the single-mode trapezoid polymer waveguide can have wider width and larger thickness than the rectangular waveguide. We believe that the trapezoid polymer waveguide may become an attractive choice as more advancements are made in developing polymers with better characteristics.

\section{ACKNOWLEDGMENT}

The authors would like to thank Dr. Ruey-Beei Wu and Dr. Way-Seen Wang for useful discussions and assistance. This work was supported in part by the National Science Council under Contract Nos. NSC86-2215-E-129-002 and NSC862215-E-002-028.

\section{REFERENCES}

1. L. D. Hutcheson and P. Hangen, "Optical Interconnects Replace Hardwire,” IEEE Spectrum, Vol. 1, 1987, pp. 30-35. 
2. R. Selvaraj, H. T. Lin, and J. F. Mcdonald, "Integrated Optical Waveguide in Polymide for Wafer Scale Integration," IEEE J. Lightwave Technol., Vol. LT-6, 1988, pp. 1034-1044.

3. R. A. Nordin, F. J. Levi, R. N. Nottenburg, J. O'gorman, T. Tanbunek, and A. R. Logan, "A System Perspective on Digital Interconnection Technology," IEEE J. Lightwave Technol., Vol. LT-10, 1992, pp. 811-827.

4. B. M. A. Rahman and J. B. Pavies, "Finite-Element Analysis of Optical and Microwave Waveguide Problem," IEEE Trans. Microwave Theory Tech., Vol. MTT-32, 1984, pp. 20-28.

5. M. Koshiba and K. Inoue, "Simple and Efficient Finite Element Analysis of Microwave and Optical Waveguides," IEEE Trans. Microwave Theory Tech., Vol. MTT-40, 1992, pp. 371-377.

6. M. N. O. Sadiku, Numerical Techniques in Electromagnetics, CRC Press, Boca Raton, FL, 1992, pp. 259-266.

(C) 1997 John Wiley \& Sons, Inc. CCC 0895-2477/97

\section{A GAIN-ENHANCED PROBE-FED MICROSTRIP PATCH ANTENNA OF VERY HIGH PERMITTIVITY}

\author{
Y. P. Zhang, ${ }^{1}$ Y. Hwang, ${ }^{1}$ and G. X. Zheng ${ }^{2}$ \\ ${ }^{1}$ Department of Electronic Engineering \\ City University of Hong Kong \\ Kowloon, Hong Kong \\ ${ }^{2}$ Institute of Confined Space Communications \\ Taiyuan University of Science and Technology \\ Shanxi, People's Republic of China
}

Received 3 December 1996; revised 17 January 1997

ABSTRACT: A probe-fed microstrip patch antenna in a very high permittivity substrate-superstrate configuration has been experimentally investigated at $1.8 \mathrm{GHz}$. The microstrip patch is much smaller and has about the same gain as a conventional microstrip patch. (c) 1997 John Wiley \& Sons, Inc. Microwave Opt Technol Lett 15: 89-91, 1997.

Key words: microstrip antenna; high-permittivity materials

\section{INTRODUCTION}

In recent years low-loss temperature-stable high-relative-permittivity $\left(\varepsilon_{r}=37,80\right)$ materials have received attention because they play a role in miniaturizing rectangular microstrip patch antennas for personal communication systems $[1,2]$. It has been shown that a direct application of a high-relativepermittivity material to reduce the dimensions of a rectangular microstrip patch antenna is accompanied by a substantial reduction in antenna bandwidth and gain. As indicated in [3], with a substrate of permittivity of 100 , the dimensions of the patch were reduced by a factor of 10 , but the radiation efficiency was reduced to $12 \%$ for a bandwidth of $0.5 \%$. Therefore the enhancement of the bandwidth and gain of microstrip patch antennas of high permittivity is highly desirable. Perrotta and Hoofar have improved the bandwidth by cutting two parallel slots in their rectangular microstrip patch on a high-permittivity substrate and optimizing the locations of the slots [2]. Here we have enhanced the gain of a microstrip patch on a high-permittivity $\left(\varepsilon_{r}=38\right)$ substrate by adding two layers of high permittivity $\left(\varepsilon_{r}=38,80\right)$ superstrates [4-6]. It is shown that a more than $10-\mathrm{dB}$ gain can be achieved.

\section{ANTENNA CONFIGURATION}

The configuration of the microstrip patch antenna is shown in Figure 1. The rectangular microstrip patch is on a very high permittivity $\left(\varepsilon_{r}=38\right)$ substrate, and is covered by two layers of superstrates of different high-permittivity $\left(\varepsilon_{r}=38,80\right)$ materials. The dimensions of the rectangular microstrip patch are $A$ and $B$. The thickness of the substrate and superstrates one and two are $t_{1}, t_{2}$, and $t_{3}$, respectively. The rectangular microstrip patch antenna is excited by a probe at $\left(x_{p}, y_{p}\right)$.

Three rectangular microstrip patch antennas designed at $1.8 \mathrm{GHz}$ are listed in Table 1. The first two single-layer antennas are used as references. The third antenna is the gain-enhanced one, which has two layers of superstrates of different high-permittivity $\left(\varepsilon_{r}=38,80\right)$ materials. The thickness of two superstrates is $1.34 \mathrm{~mm}\left(0.04960 \lambda_{2}\right)$ and $3.35 \mathrm{~mm}$ $\left(0.1799 \lambda_{3}\right)$, respectively. Compared with the size of the first reference antenna, the sizes of the patches of the second reference antenna and the gain-enhanced antenna are approximately reduced by 9.7 and 11.45 times, respectively.

\section{EXPERIMENTAL RESULTS}

Figure 2 shows the measured return loss as a function of frequency. The dash-dot curve depicts the measured return loss of the gain-enhanced antenna. The solid and dashed curves display the measured return losses of reference antenna one and two, respectively. It is seen that good matching has been achieved for all three antennas. The antennas are resonant at $1.8 \mathrm{GHz}$. Using $\left|S_{11}\right| \leq-10$ as a criterion, the measured impedance bandwidths are $19.5 \mathrm{MHz}(1.083 \%)$ for the first reference antenna, $19.5 \mathrm{MHz}(1.083 \%)$ for the second reference antenna, and $16.5 \mathrm{MHz}(0.917 \%)$ for the gain-enhanced antenna.

Figures 3(a) and 3(b) show the copolarized E- and H-plane radiation patterns of three antennas, respectively. The upper and lower continuous curves in Figure 3(a) represent the relative received power by reference antennas one and two. The corresponding maximum relative received powers are $-32.2 \mathrm{dBm}$ and $-43.7 \mathrm{dBm}$. The impact of adding two superstrates with optimal thickness can be seen from the

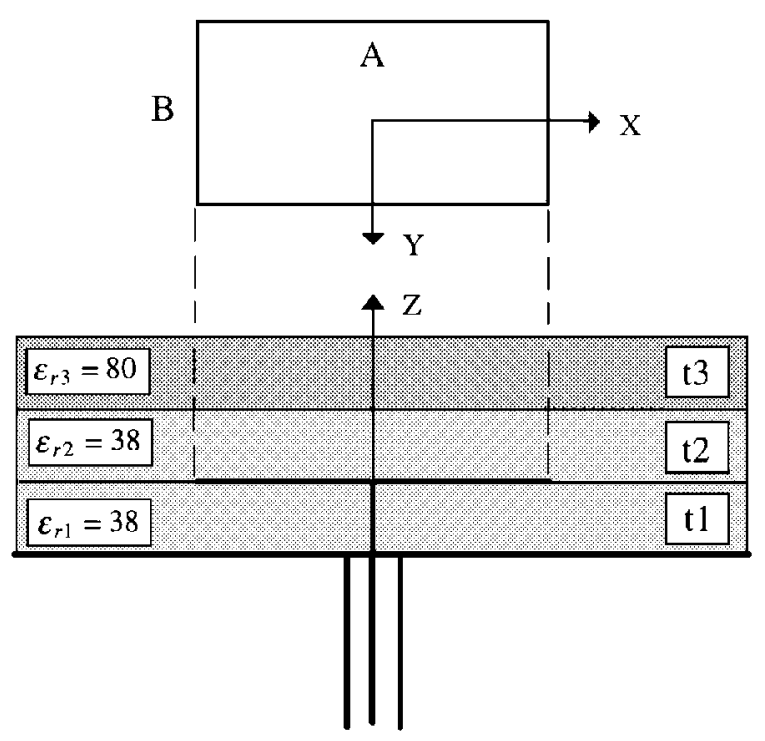

Figure 1 Geometry of the gain-enhanced rectangular microstrip patch antenna 